\title{
The Use of Mode Components for Calculating Steady-state Processes in Long or Short Multiphase Lines
}

\author{
Ivars Dumins, Riga Technical University
}

\begin{abstract}
The calculation of steady-state processes in the nonsymmetric multiphase long or short line by using the distribution of the solution for the voltages differential equations in mode components is considered. The matrices of integrations constants can be obtained if the voltages or the currents at the beginning of the line and the matrices of input are known. The elements of these matrices are to be calculated directly from the definition. The distribution of the voltages along the specific 4-wire line is calculated. The fault of the phase to neutral is calculated in a specific 4-wire line. The method can be recommended for the calculation of short lines too.
\end{abstract}

Keywords - non-symmetrical multiphase line; matrix; transposition; fault.

\section{INTRODUCTION}

The solving of differential equations of the multiphase line by distribution of the solution in mode components has been known since 1960-s [1], however this method has been applied mainly for investigating transient processes in lossless lines [2] - [8]. In these cases active losses have been kept by switching lumped resistances at the terminals of calculations schema. In steady-state processes, however, it is possible to consider quite accurately each of the primary parameters of the symmetrical or the non-symmetrical long or short line.

\section{MATHEMATICAL BASIS}

The differential equation for vector of complex voltages $\boldsymbol{U}$ is the following:

$$
\frac{d^{2} \boldsymbol{U}}{d x^{2}}=(\boldsymbol{R}+j \omega \boldsymbol{L})(\boldsymbol{G}+j \omega \boldsymbol{C}) \boldsymbol{U}=\boldsymbol{Z} \boldsymbol{Y} \boldsymbol{U},
$$

where $\boldsymbol{R}, \boldsymbol{L}, \boldsymbol{G}, \boldsymbol{C}$ are the square matrices of primary parameters, $\omega, x, j$ are the given frequency, the coordinate along the line and the imaginary unit. If the number of the wires is $n+1$ all these matrices have the order $n . R, L, G, C$ as well as $\boldsymbol{Z}=\boldsymbol{R}+j \omega \boldsymbol{L}$ and $\boldsymbol{Y}=\boldsymbol{G}+j \omega \boldsymbol{C}$ are symmetric matrices if the elements of the vector $\boldsymbol{U}$ are the phase voltages even if the placement of the wires in the space is not symmetrical. (These matrices can be nonsymmetrical if the line voltages are the elements of the vector $\boldsymbol{U}$, but it does not mean that the principle of reciprocity would not be correct.)

Matrix $\boldsymbol{Z} \boldsymbol{Y}$ can be written as $\boldsymbol{T} \boldsymbol{\Lambda} \boldsymbol{T}^{-1}$, where $\boldsymbol{\Lambda}$ is a diagonal matrix of $\boldsymbol{Z Y}$ eigenvalues $\lambda_{1} \ldots \lambda_{n}, \boldsymbol{T}$ is the square matrix of $\boldsymbol{Z Y}$ eigenvectors. By substituting this in (1) and pre-multiplying the equation by $\boldsymbol{T}^{-1}$ we obtain the following equation

$$
\frac{d^{2} \boldsymbol{U}_{\mathrm{mod}}}{d x^{2}}=\boldsymbol{\Lambda} \boldsymbol{U}_{\mathrm{mod}},
$$

where $\boldsymbol{U}_{\text {mod }}=\boldsymbol{T}^{-1} \boldsymbol{U}$ is the vector of the mode components.

Since $\Lambda$ is a diagonal matrix the matrix-equation (2) splits into separate equations for each of the components and their solution is

$$
U_{\text {mod }, k}=B_{1 k} \operatorname{ch} \gamma_{k} x+B_{2 k} \operatorname{sh} \gamma_{k} x,
$$

where $\gamma_{k}=\sqrt{\lambda_{k}}$ is the propagation constant of the mode component $k, B_{1 k}$ and $B_{2 k}$ are the constants of integration.

We can write the solution of (2) in matrix form by combining left and right sides of all the expressions (3) in vectors:

$$
\boldsymbol{U}_{\text {mod }}=\mathbf{C h}(x) B_{1}+\mathbf{S h}(x) B_{2},
$$

where $\boldsymbol{B}_{\mathbf{1}}$ and $\boldsymbol{B}_{\mathbf{2}}$ are the vectors of constants, but $\mathbf{C h}(x)$ and $\operatorname{Sh}(x)$ are the diagonal matrices with elements $\operatorname{ch} \gamma_{k} x$ and $\operatorname{sh} \gamma_{k} x$.

Since $\boldsymbol{U}=\boldsymbol{T} \boldsymbol{U}_{\text {mod }}$ we can write the solution of (1) for the vector of true voltages too [9]:

$$
\boldsymbol{U}(x)=\boldsymbol{T}\left(\mathbf{C h}(x) B_{1}+\mathbf{S h}(x) B_{2}\right) .
$$

The vector of currents $\boldsymbol{I}$ and the voltages $\boldsymbol{U}$ are linked by the expression

$$
\boldsymbol{I}=-\boldsymbol{Z}^{-1} d \boldsymbol{U} / d x
$$

which allows obtaining the solution for $\boldsymbol{I}$ :

$$
\boldsymbol{I}(x)=-\boldsymbol{Z}^{-1} \boldsymbol{T} \Gamma\left(\mathbf{S h}(x) B_{1}+\mathbf{C h}(x) B_{2}\right),
$$

where $\Gamma$ is the diagonal matrix with the elements $\gamma_{\mathrm{k}}$. (Thus we have been freed from the need to look at the individual mode components).

The finding of the constants of integration depends on what is known in the line. If the voltages $\boldsymbol{U}(0)$ at the point $x=0$ (for example, at the beginning of the line) are given from (4) we obtain

$$
\boldsymbol{B}_{\mathbf{1}}=\boldsymbol{T}^{-1} \boldsymbol{U}(0) .
$$

If at the terminal of the line $(x=l)$ a passive multipole is connected the matrix $\boldsymbol{y}$ of input and mutual admittances of which are known then on one hand

$$
\boldsymbol{I}(l)=\boldsymbol{y} \boldsymbol{U}(l)=\boldsymbol{y} \boldsymbol{T}\left[\left(\mathbf{C h}(l) B_{1}+\mathbf{S h}(l) B_{2}\right)\right],
$$

but on the other hand from (5)

$$
\boldsymbol{I}(l)=-\boldsymbol{Z}^{-1} \boldsymbol{T} \Gamma\left[\mathbf{S h}(l) B_{1}+\mathbf{C h}(l) B_{2}\right] .
$$

If vector $\boldsymbol{B}_{\mathbf{1}}$ is found then it is possible to find $\boldsymbol{B}_{\mathbf{2}}$ too by equating the last two expressions:

$\boldsymbol{B}_{2}=-\left[\boldsymbol{y} \boldsymbol{T} \mathbf{S h}(l)+Z^{-1} \boldsymbol{T} \Gamma \operatorname{Ch}(l)\right]^{-1}\left[Z^{-1} \boldsymbol{T} \Gamma \operatorname{Sh}(l)+y \boldsymbol{T} \mathbf{C h}(l)\right] B_{1}$. (8)

In the other case when the currents at the beginning of the line $\boldsymbol{I}(0)$ are given first we can obtain from (5) 


$$
\boldsymbol{B}_{2}=-\left(\boldsymbol{Z}^{-1} \boldsymbol{T} \Gamma\right)^{-1} \boldsymbol{I}(0),
$$

then from (7) $\boldsymbol{B}_{1}$ too:

$$
\boldsymbol{B}_{1}=-\left[\boldsymbol{y} \boldsymbol{T} \operatorname{Ch}(l)+Z^{-1} \boldsymbol{T} \Gamma \operatorname{Sh}(l)\right]^{-1}\left[\boldsymbol{y} \boldsymbol{T S h}(l)+Z^{-1} \boldsymbol{T} \Gamma \operatorname{Ch}(l)\right] B_{2} .
$$

(It is not necessary to attempt to simplify such matrix expressions: if the order of matrices $n$ is not very large then there is no difficulty to calculate the necessary products or the inverse matrices by using suitable mathematical programs).

After the determination of constants it is possible to calculate voltages and currents at any place of the homogeneous line by using expressions (4) and (5).

If the line consists of several homogeneous sections with different initial parameters then it is possible to calculate the input matrix $\boldsymbol{y}_{\mathbf{1}}$ of the last section by using the given matrix $\boldsymbol{y}$ of the load and (7), (8), (5). The elements of this matrix are to be calculated directly from definition - as a ratio of the current to the voltage in cases when only one element in the vector $\boldsymbol{U}(0)$ is not equal to zero. (The count of coordinate in each section begins with a zero.) When calculating elements in the first row of $\boldsymbol{y}_{\mathbf{1}}$ in the vector $\boldsymbol{U}$ the first element is different from zero, for the second row - the second element etc. A non-zero element of $\boldsymbol{U}$ can be equal to 1 , then the elements of $\boldsymbol{y}_{\mathbf{1}}$ are equal to the relevant currents calculated. The calculated $\boldsymbol{y}_{1}$ works as the "load" matrix for the penult section etc. until the "load" matrix of the first section is obtained. Thereafter it is possible to calculate voltages and currents in the first section by using the true matrix $\boldsymbol{U}(0)$. The values of the voltages at the end of the first section $\boldsymbol{U}\left(l_{1}\right)$ can be used as $\boldsymbol{U}(0)$ for the second section etc. This way, for example, we can take into account every transposition of the wires, but by projecting a new line we can choose the best kind of transpositions.

\section{EXAMPLE 1: TRANSPOSITIONS IN LINE}

A non-symmetric 4-wire line was taken as an example (fig. 1). (Instead of usual symbols A,B,C,0 for three-phase lines the wires are numbered. This makes the operations with the matrices much easier.) The distances between the wires were $d_{12}=1 \mathrm{~m} ; d_{23}=2 \mathrm{~m} ; d_{34}=4 \mathrm{~m}$; the radius of all the wires was $r_{0}=5 \mathrm{~mm}$; the resistances of every wire were $R_{1}=R_{2}=R_{3}=R_{4}=0.25 \Omega / \mathrm{km}$; the directions of the currents in wires $1,2,3$ were selected the same, in wire 4 - the opposite.

Magnetic fluxes through the areas (with the length $d x$ ) formed by each of the wires 1,2,3 with wire 4 can be expressed taking into account the directions of the currents. Then matrix $\boldsymbol{L}$ is obtained:

$$
\boldsymbol{L}=\left(\begin{array}{ccc}
2 l_{14} & l_{24}-l_{12}+l_{41} & l_{34}-l_{13}+l_{41} \\
m_{124}+l_{42} & 2 l_{24} & l_{34}-l_{32}+l_{42} \\
m_{134}+l_{43} & m_{234}+l_{43} & 2 l_{34}
\end{array}\right),
$$

where $\left.l_{i k}=l_{k i}=2 \cdot 10^{-4}\left[\ln \left(d_{i k} / r_{0}\right)+0.25\right)\right](\mathrm{H} / \mathrm{km})$ is inductance of circuit formed by the wires $i$ and $k, m_{i j k}$ is the mutual inductance between the wire $i$ and circuit of the wires $j$ and $k$. Matrix $\boldsymbol{L}$ is symmetric, i.e. $m_{124}+l_{42}=l_{24}-l_{12}+l_{41}$ etc. (The influence of the earth here is not considered.)
Similarly, we can write matrices $\boldsymbol{R}, \boldsymbol{C}, \boldsymbol{G}$ taking into account the active drop of voltage in the wires and the leakage currents between the wires:

$$
\begin{gathered}
\boldsymbol{R}=\left(\begin{array}{ccc}
R_{1}+R_{4} & R_{4} & R_{4} \\
R_{4} & R_{2}+R_{4} & R_{4} \\
R_{4} & R_{4} & R_{3}+R_{4}
\end{array}\right) ; \\
\boldsymbol{C}=\left(\begin{array}{ccc}
c_{12}+c_{13}+c_{14} & -c_{12} & -c_{13} \\
-c_{12} & c_{12}+c_{23}+c_{24} & -c_{23} \\
-c_{13} & -c_{23} & c_{13}+c_{23}+c_{34}
\end{array}\right) .
\end{gathered}
$$

Values of $c_{\text {ik }}$ in this case were chosen so that the speed of the waves of the transient process depending on the eigenvalues of the matrix $\boldsymbol{L} \boldsymbol{C}$ could not exceed the speed of the light, but they can be calculated more precisely by using the placement of the wires in the space. $\boldsymbol{G}$ was proportional to the $\boldsymbol{C}$ to the aspect ratio of $10^{3}$.

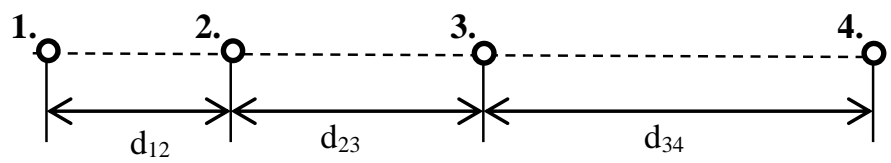

Fig. 1. Placement of wires for the line considered.

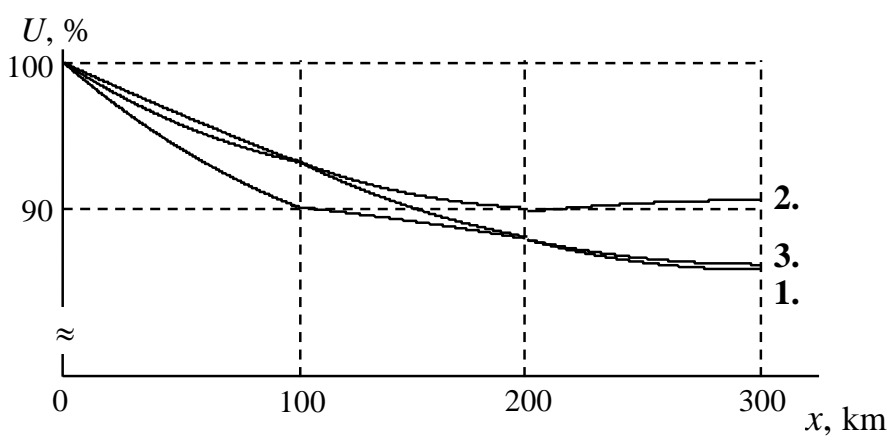

Fig. 2. Distribution of phase voltages along the line (2 transpositions).

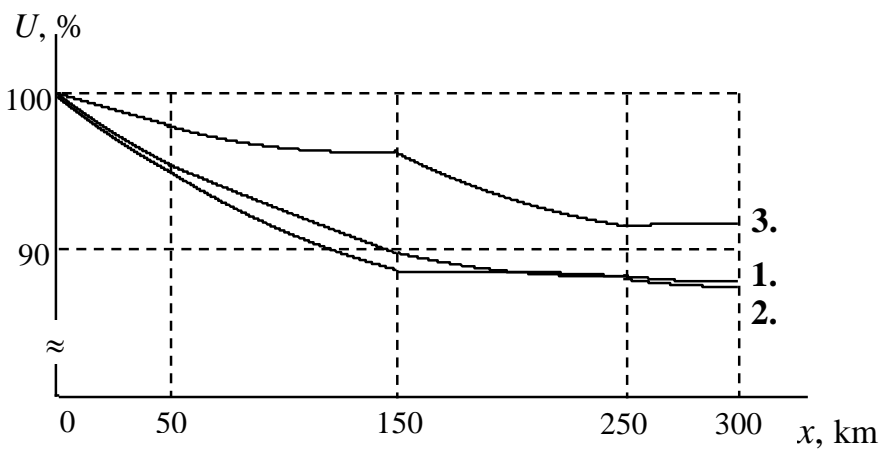

Fig. 3. Distribution of phase voltages along the line (3 transpositions).

The distribution of the modules of voltage along the line was calculated for two cases with this method. First, the length of the line $l=300 \mathrm{~km}$ was divided into 3 equal sections by two transpositions (fig. 2), then into 4 sections of length $l / 6, l / 3, l / 3$, $1 / 6$ by three transpositions (fig. 3 ). A symmetric system of threephase voltage was connected to the beginning of the line. There was no-load at the end. 
It is evident that the difference between the phase voltages at the terminal reaches $7 \%$ of the beginning voltage if there are only two transpositions. Three transpositions reduce it to $4 \%$, but the line cannot be called fully symmetrical anyway. (The presence of the load could cause a reduction in the difference. In the absence of transpositions differences could achieve $30 \%$ in such line).

\section{EXAMPLE 2: THE FAULT OF PHASE TO NEUTRAL}

Some difficulties can occur if we must consider the presence of different lumped elements (for example, resistances) in the multiphase line. That makes us split the line into separate homogeneous sections. Unfortunately we have no general theory of multipoles that would easily calculate the change of the matrix $\boldsymbol{y}$ when additional lumped elements are connected to it.

We can use the double-T diagram of 4-pole (in generalized meaning when currents are not equal in pairs) when we consider a 4-wire line [10]. The diagram consists of 6 impedances respective to six different elements of a symmetric matrix of order 3 (Fig. 4.). We can calculate the impedances $Z_{1} \ldots Z_{6}$ if the matrix of the admittances $\boldsymbol{y}$ is known:

$$
\begin{array}{ll}
Z_{1}=-y_{23} / P, & Z_{4}=\left(y_{11}+y_{12}+y_{13}\right)^{-1} ; \\
Z_{2}=-y_{13} / P, & Z_{5}=\left(y_{12}+y_{22}+y_{23}\right)^{-1} ; \\
Z_{3}=-y_{12} / P, & Z_{6}=\left(y_{13}+y_{23}+y_{33}\right)^{-1},
\end{array}
$$

where $P=y_{12} y_{23}+y_{12} y_{13}+y_{23} y_{13}$.

Then we can connect to the diagram the elements needed and calculate the new matrix $\boldsymbol{y}$ at least by Kirchhoff's laws. For example, we can include in series the great resistance $\left(Z_{7}\right)$ with the terminal 2 by modeling the break of wire 2 or small resistance $\left(Z_{8}\right)$ between 2 and 0 by modeling the fault of phase 2 to neutral.

In Fig. 5 the change of phase voltages along the line with the length $100 \mathrm{~km}$ is shown when in the middle of the line a fault of wire 2 to neutral occurs. The load of the line was starconnection of 3 equal resistances of $400 \Omega$ (it approximately corresponds to the transmitted power of $3 \mathrm{MW}$ at the nominal voltage of $20 \mathrm{kV}$ ). The line is not long and the change of voltages is approximately linear. The voltage of the phase 2 , of course, decreases to zero at the fault point in the middle of the line and then it restores to approximately $20 \%$ of the beginning voltage across the load. The voltage of the phases 1 and 3 , on the contrary, increase reaching an overvoltage of $20 \%-30 \%$ at the points near the fault. (That agrees well with the known data [11-13].) Some overvoltage remains in the phase 1 and 3 till the terminal of the line. The current in the wire 2 in the first section of the line is limited only by impedance of the wires; it is very large (our method allows calculating it too). The magnetic field of it obviously causes the appearance of overvoltages in the phases 1 and 3 .

\section{V.CONCLUSION}

The use of different, not always entirely correct simplifications such as the replacement of the multiphase line by the two-wire line, concepts of averaged inductance or mutual inductance of "phase" etc. may refuse using this

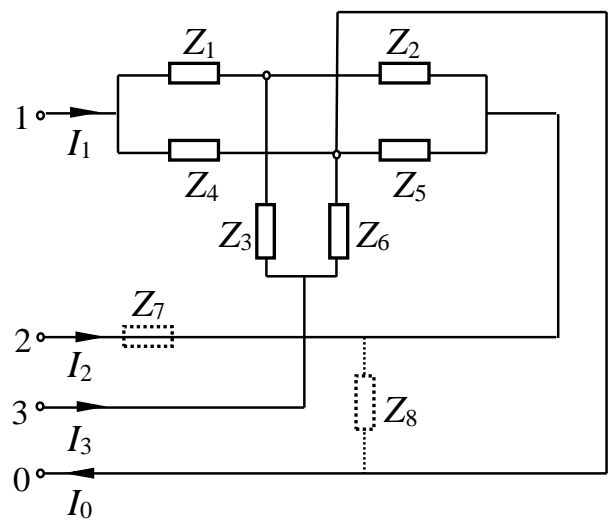

Fig. 4. It is possible to model faults in the 4-wire line by connecting in series great impedance to equivalent diagram of 4 -pole $\left(Z_{7}\right.$ - break of wire 2$)$ or small impedance between wires ( $Z_{8}$ - fault of wire 2 to neutral).

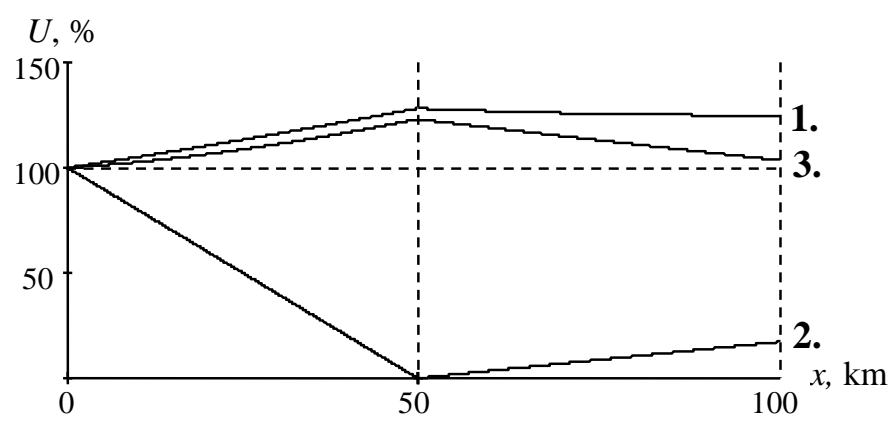

Fig. 5. The change of phase voltages in the line on the fault of phase 2 to neutral.

method. The separation of the homogeneous line into separate sections which are then replaced by an equivalent circuit which can contain even 20 or more lumped elements is totally unnecessary.

The method is simple and would be recommended for the calculation of short lines too (such as that in fig. 5). Accuracy, as always, depends on that of the primary parameters.

In the future this method should be combined with the method of symmetrical components to account for the effect of rotating machines.

\section{REFERENCES}

[1] H. W. Dommel, "Digital computer solution of electromagnetic transient in single and multiphase networks," IEEE Transactions on Power Apparatus and Systems, vol. PAS-88, no. 4, pp. 388-397, 1969. http://dx.doi.org/10.1109/TPAS.1969.292459

[2] H. W. Dommel, W. S. Meyer, "Computation of electromagnetic transients", Proc. IEEE, vol. 62, no. 7, pp. 983-993, 1974. http://dx.doi.org/10.1109/PROC.1974.9550

[3] H.W. Dommel, Electromagnetic transients program reference manual (EMTP theory book). Bonneville Power Administration, Oregon, 1986.

[4] H. W. Dommel, EMTP Theory Book, Microtran Power System Analysis Corporation, Canada, 1992.

[5] D. A. Woodford, "Validation of digital simulation of DC links," IEEE Trans. Power Apparatus and Systems, vol. PAS-104, no. 9. 1985. http://dx.doi.org/10.1109/TPAS.1985.319024

[6] D. Brandt, R. Wachel, R. Valiquette, "Closed loop testing of a joint VAR controller using a digital real-time simulator," IEEE Trans. On Power Systems, vol. 6, no. 3, 1991. http://dx.doi.org/10.1109/59.119258

[7] J. A. Brandeo Faria, J. Hildemaro Bricero, "On the modal analysis of asymmetrical three-phase transmission lines using standard transformations", IEEE Trans. Power Delivery, vol. 12, no. 4, pp. 1760-1765, 1997. http://dx.doi.org/10.1109/61.634202 
[8] A. Haddad, D. Warne, (ed.) Advances in high voltage engineering. The Institution of Engineering and Technology, London UK, pp. 316-318, 2007

[9] I. Dūminuš̆, Elektrotehnikas teorētiskie pamati. Pārejas procesi, garās līnijas un nelineārās kèdes, Rīga, Zvaigzne ABC, 2006, 239-245 lpp.

[10] Z. Broka, I. Dumins, "Multipole as an element of electric circuit and its equivalent diagrams," Scientific Journal of Riga Technical University. Power and electrical engineering, ser. A, vol. 28, pp. 68-71, 2011.

[11] W. Diesendorf, "Temporary overvoltages," in High-volage elektric power systems, Butterworths, London, 1974.

[12] CIGRE working group and IEEE force on TOV, „Temporary overvoltages, causes, effects and evaluation", in CIGRE conference 1990 session, pp. 33-210, 1990.

[13] A. Haddad, D. Warne (ed.), Advances in high voltage engineering, The Institution of Engineering and Technology, London UK, pp. 318-320, 2007. http://dx.doi.org/10.1049/PBPO040E
Ivars Dumins ed.: University of Latvia, Riga Politechnical Institute, Moscow Power-Engineering institute. Dipl. ing. (Riga, 1960), cand. techn. sc. (Moscow, 1969), Dr. sc. ing. (Riga, 1992).

Worked in Riga Electromachine-building Plant, Riga Polytechnic Institute, Moscow Power-Engineering Institute, Riga Technical University (Professor, 1997). Sc. works in magnetic hydrodynamics, nuclear fusion engineering challenges, theory of electrical circuits and electromagnetic field. Author or co-author of 5 basic electrical engineering text-books.

Member of the Latvian Association of University and College Professors. E-mail: ivars.dumins@rtu.lv, ivd@apollo.lv 